remaining normal main lacrimal gland acini. The cyst size and its rapid reaccummulation following aspiration supports this theory of its origin since the main lacrimal gland produces tears at a rate that can exceed $50 \mu 1 / \mathrm{min}^{4}$ as opposed to the basic secretion from the glands of Kraus and Wolfring that produces less than $0.3 \mu 1 / \mathrm{min}^{5}$ In addition to ductal occlusion secondary to conjunctival scarring, we postulate that the inflammation around the ducts as seen histopathologically may have caused the ductal walls to weaken and more readily become ectatic forming this giant cyst.

Complete cyst excision by the transconjunctival approach is usually recommended. ${ }^{1}$ In this particular patient, aside from the difficulty of everting the lid to approach the cyst subconjunctivally, violating the conjunctiva in the setting of OCP is not advisable. Manipulation of the conjunctiva may provoke an acute flare of disease activity as observed in a previous report. ${ }^{3}$ The lid crease approach also provided better visualisation of the levator aponeurosis dehiscence which could be repaired primarily. Simple aspiration is not recommended since this will result in immediate recurrence.

We thank Michael Warner, MD, for histopathology photomicrographs.

HEIDI D REMULLA Eye Plastics and Orbit Service, Massachusetts Eye and Ear Infirmary Harvard Medical School

Boston, USA

Correspondence to: Peter AD Rubin, MD,

Director, Eye Plastics and Orbital Surgery,

Massachusetts Eye and Ear Infirmary, 243 Charles

Street, Boston, MA 02114, USA.

Accepted for publication 18 July 1995

1 Duke-Elder S, ed. System of ophthalmology. Vol XIII: The ocular adnexa. Part II: Lacrimal, orbital and para-orbital diseases. St Louis: Mosby, 1974. 638-43.

2 Weatherhead RG. Wolfring dacryops. Ophthalmology 1992; 99: 1575-81.

3 Mondino BJ, Brown SI, Lempert S, Jenkins MS The acute manifestations of ocular cicatricial pemphigoid: diagnosis and treatment. Ophthalmology 1979; 86: 543-51.

4 Jordan A, Baum J. Basic tear flow. Does it exist? Ophthalmology 1980; 87: 920-30.

5 Fullard RJ, Snyder C. Protein levels in nonstimulated and stimulated tears of normal human subjects. Invest Ophthalmol Vis Sci 1990; 31: 1119-26.

\section{Angiolymphoid hyperplasia with eosinophilia (Kimura's disease) of the conjunctiva}

EDITOR,-Kimura's disease is a rare chronic vascular inflammatory disorder occurring predominantly in orientals, with occasional cases in white and black people. ${ }^{1}$ The disease first appeared as 'eosinophilic hyperplastic lymphadenopathy' in the Chinese literature, but the disorder is commonly known as Kimura's disease, following the report of Kimura $e t$ al in the Japanese literature. ${ }^{2}$

Kimura's disease predominantly involves the head and neck region, and orbital involvement has been reported. ${ }^{1}$ The lesions may be isolated or multiple and vary in clinical appearance.

Histopathologically, the lesions are composed of vascular hyperplasia with plump endothelial cells accompanied by varying degrees of mixed cellular infiltrates dominated by lymphocytes and eosinophils. ${ }^{3}$

Conjunctival Kimura's disease, to our knowledge, has not been described in the English language published reports.
CASE REPORT

A 24-year-old white girl presented in March 1994 with a painless ulcerated white lesion of the bulbar conjunctiva of the right eye. There was no history of trauma. She was otherwise in good health.

On examination visual acuity was $6 / 4$ bilaterally. An ulcerated, mobile firm swelling was noted in the inferotemporal aspect of the bulbar conjunctiva, with no associated lymphadenopathy. Systemic examination was normal.

Routine investigations for full blood count, sedimentation rate, syphilis serology, and angiotensin converting enzyme were normal. Serum concentrations of immunoglobulin IgA and IgM were elevated. The peripheral blood eosinophil level was normal.

Conjunctival scrapings for bacteria, viruses, chlamydia, and fungi were negative A chest radiograph was also normal. Computed tomography demonstrated an anterior orbital soft tissue swelling with no evidence of a foreign body within the lesion Treatment with topical steroids and systemic antibiotics led to no improvement in the clinical condition.

The lesion became increasingly uncomfortable, and extended onto the inferior tarsal conjunctiva. Figure 1 shows the yellow-white nodular mucosal plaque measuring $15 \mathrm{~mm}$ by $10 \mathrm{~mm}$. Biopsy and debulking of the lesion was performed. The histopathological examination revealed a tan-coloured nodule which measured $15 \times 10 \times 7 \mathrm{~mm}$. Ulceration of the conjunctiva was present with underlying prominent vascular inflammatory stroma. The base of the ulcer showed inflammatory granulation tissue and included neutrophils, eosinophils, and lymphocytes. Lymphoid aggregates with reactive germinal centres were also present (Fig 2 top). Abundan blood vessels lined by plump endothelial cells and surrounded by lymphocytic and eosinophilic infiltration were also prominen features (Fig 2 bottom). Histiocytic granulomata were not seen. Special stains showed no organisms within the ulcer. The histopathological appearance was that of angiolymphoid hyperplasia with eosinophilia.

After a'course of topical steroids there was no change in the residual lesion and furthe debulking with intralesional steroids was performed.

\section{COMMENT}

Kimura's disease is an inflammatory process of unknown aetiology, with a predilection for the head and neck region. Controversy exists as to whether it represents an allergic response to a continuing antigenic stimulus or a benign lymphoid neoplastic process Lymphadenopathy, peripheral blood eosinophilia, and elevated serum immunoglobulin IgE are features of Kimura's disease. ${ }^{2}$ In this

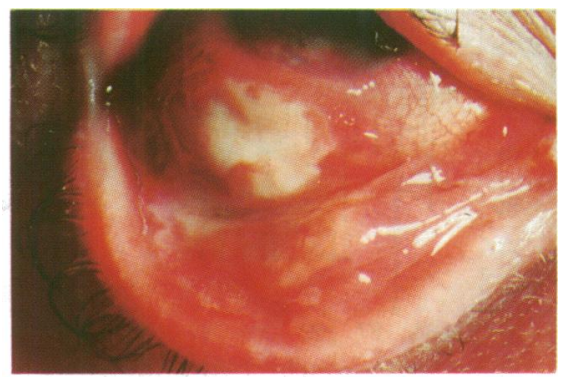

Figure 1 Kimura's disease of the bulbar conjunctiva extending onto the tarsal conjunctiva. patient an elevated serum immunoglobulin IgA and IgM was noted. Systemic associations include asthma and nephrotic syndrome. ${ }^{3}$ The long term natural history of the disease is uncertain. Whether Kimura's disease and angiolymphoid hyperplasia with eosinophilia represent two distinct clinicopathological disorders, ${ }^{4}$ or different appearances of the same disease is still debated and is beyond the scope of this report.

Kimura's disease may present as a solitary or multiple lump(s) of insidious onset. Orbital disease may be preceded by other foci of involvement, such as the parotid gland. ${ }^{4}$ The first orbital case was recorded in 1976, and in 1983 , a series of five orbital and three adnexal cases was published. ${ }^{3}$ The mean age of patients with orbital disease was higher than the mean age at other sites. The lesions were solitary, and the location of the lesions varied from the eyebrow to the roof of the orbit. Kimura's disease occurring in association with an Iowa enucleation implant has also been reported. ${ }^{5}$ Conjunctival Kimura's disease has not been reported in the English language published reports.

The histopathological features of Kimura's disease include endothelial cell proliferation, eosinophilic infiltration, lymphoid aggregates with florid germinal centres, fibrous sclerosis, and giant cells of the Warthin-Finkeldy type

The pathological differential diagnosis of Kimura's disease includes inflammatory pseudotumour but the eosinophils in these cases are not usually such a conspicuous feature. Other disease processes such as Hodgkin's disease can be differentiated by the presence of Reed-Sternberg cells and bacillary angiomatosis by proliferating endothelial cells in association with clumps of bacteria.

Previously reported cases of periorbital Kimura's disease were successfully treated with excisional biopsy, though excessive bleeding during the surgery was noted. ${ }^{3}$ The systemic use of corticosteroids in orbital disease is also recognised though there may be recurrence. ${ }^{6}$

Skin disease has been successfully treated with intralesional triamcinolone, carbon

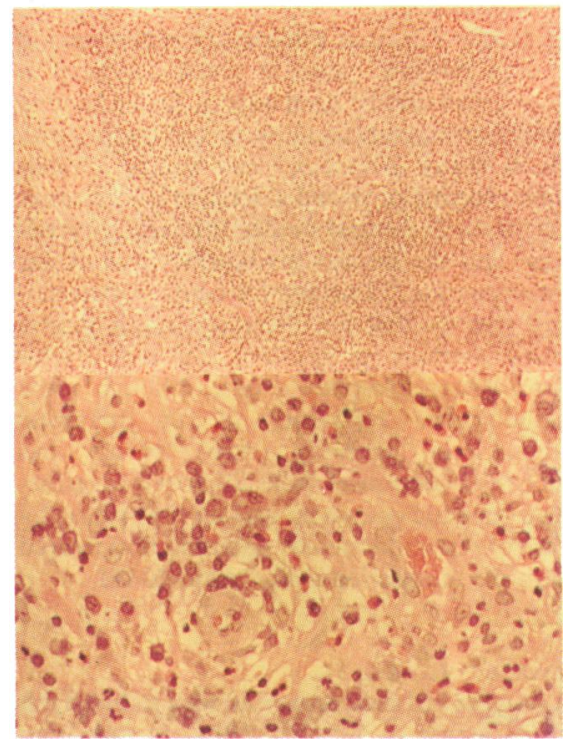

Figure 2 (Top) Haematoxylin and eosin stain showing lymphoid aggregate with a reactive germinal centre surrounded by inflammatory stroma (magnification $\times 85$ ). (Bottom) Haematoxylin and eosin stain showing blood vessels with plump endothelial cells and surrounding mixed cellular infiltration including lymphocytes and eosinophils (magnification $\times 170$ ). 
dioxide laser, and retinoic acid; some success has also been reported with radiotherapy. ${ }^{3}$

Kimura's disease probably represents a reactive inflammatory process to an undetermined antigenic stimulus. Association with increased serum IgE levels, and in our patient both IgA and IgM levels, support a reactive origin for this disease.

We thank Professor W R Lee for his help and advice on the pathology.

D G R JAYAMANNE $S$ K WEBBER REW RIDLEY D G COTTRELL Department of Ophthalmology, Newcastle General Hospital, Newcastle upon Tyne J H MCCARTHY South Tyneside District Hospital, Tyne and Wear

Correspondence to: Mr D G R Jayamanne, Department of Ophthalmology, Newcastle General Hospital, Westgate Road, Newcastle upon Tyne NE4 6BE.

Accepted for publication 27 June 1995

1 Kennedy SUM, Pitts JF, Lee WR, Gibbons DC Bilateral Kimura's disease of the eyelids. $\mathrm{Br} \mathscr{f}$ Ophthalmol 1992; 76: 755-7.

2 Kimura T, Yoshimura S, Ishikura E. On the unusual granulation combined with hyperplastic changes of lymphatic tissue. Trans Soc Pathol fpn 1948; 37: 179-80.

3 Hidayat AA, Cameron JD, Font RL, Zimmermann LE. Angiolymphoid hyperplasia with eosinophilia (Kimura's disease) of the with eosinophilia (Kimura's disease) of the
orbit and ocular adnexa. Am $\mathcal{f}$ Ophthalmol 1983; 96: 176-89.

4 Kung ITM, Gibson JB, Bannatyne PM. Kimura's disease: a clinico-pathological study of 21 cases and its distinction from angiolymphoid hyperplasia with eosinophilia. Pathology 1984; 16: 39-44.

5 Smith DL, Kincaid MC, Nicolitz E. Angiolymphoid hyperplasia with eosinophilia (Kimura's disease) of the orbit. Arch (Kimura's disease) of the orbit. Arch Ophthalmol 1988; 106: 793.

6 Sheren SB, Custer PL, Smith ME. Angiolymphoid hyperplasia with eosinophilia of the orbit associated with obstructive airway disease. Am f Ophthalmol 1989; 108: 167-9.

Monoclonal antibody therapy of chronic intraocular inflammation using Campath-1H

EDITOR,-Chronic intraocular inflammation is an important cause of visual handicap in young and middle aged adults. Accumulating evidence suggests an autoimmune pathogenesis in many instances, ${ }^{1}$ and animal models have recently provided a detailed understanding of both normal ocular immune physiol$\mathrm{ogy}^{2}$ and the immunopathogenesis of ocular inflammation. ${ }^{1}$ Therapy for ocular inflammation mirrors that for systemic autoimmunity: corticosteroids progressing through cyclosporin A to cytotoxic drugs, although some cases prove refractory to all therapies. Monoclonal antibody (mab) therapy has recently been successfully employed in refractory systemic vasculitis ${ }^{3}$ and we now present a case of refractory ocular inflammation treated with the humanised mab Campath-1H.

CASE REPORT (Fig 1)

A 36-year-old man presented with reduced left visual acuity in October 1984. Examination revealed a panuveitis but systemic examination and all investigations were normal, including autoantibodies. Symptoms improved with prednisolone (40 mg/day) but during the next 12 months they returned whenever the dosage was reduced. In December 1985 cyclosporin A was commenced ( $5 \mathrm{mg} / \mathrm{kg} /$ day) but inflammation still recurred when the prednisolone dosage fell below $30 \mathrm{mg} /$ day. By April 1990, a panuveitis also affected the right eye and the visual acuity fell to counting fingers bilaterally. Examination revealed cells and flare in both anterior segments. The binocular indirect ophthalmoscopy (BIO) score was 3 in both eyes and multiple choroidal infiltrates were present throughout both fundi. Fluorescein angiography showed retinal vasculitis but immunological screening remained normal. The patient was now hypertensive with a diminished creatinine clearance, and azathioprine was substituted for cyclosporin A. During the next 6 months relapses continued despite the addition of cyclophosphamide (Fig 2). In July 1991 azathioprine and cyclophosphamide were stopped owing to their inefficacy and the development of a mild leucoerythroblastic anaemia. Pulses of methylprednisolone (thrice weekly for 3 months) and $20 \mathrm{mg}$ of oral prednisolone daily failed to prevent deterioration with central vasculitis leading to optic atrophy and macular ischaemia. In July 1992, a posterior subcapsular lens opacity developed and a right cataract extraction with lens implant was performed. The patient was Cushingoid with hypertension, proximal myopathy, peripheral neuropathy, vertebral collapse, and achilles tendon rupture but visual acuity continued to deteriorate when steroids were reduced with best vision of counting fingers (left) and 6/9 (right). A course of intravenous immunoglobulin (IVIG) resulted in a brief respite in May 1993 but a subsequent relapse led to consideration of Campath-1H therapy in November 1993. At that time the patient was taking only prednisolone $15 \mathrm{mg}$ daily. Visual acuity was counting fingers (right) and hand movements (left) and examination revealed vitreal cells and debris bilaterally (BIO score of 3-4) with active choroiditis and retinal vasculitis. A dose of $12 \mathrm{mg}$ of Campath$1 \mathrm{H}$ was administered daily for 5 days by intravenous infusion. There was an immediate fall in peripheral blood lymphocyte count, and subjective and objective ocular improvement was present by day 4 . Visual acuity at discharge (day 10 ) was $6 / 18$ (right) and hand movements (left) and examination revealed vitreal clearing. Prednisolone was reduced to $10 \mathrm{mg}$ daily. Two weeks after discharge there was no evidence of disease activity and vitreal BIO scores were 0 bilaterally. A minor relapse at week 12 was reversed with a temporary ( 3 days) increase in steroid dose to $30 \mathrm{mg}$. There was a further deterioration in symptoms at week 18 but this regressed spontaneously without medical intervention. Visual acuity in January 1995 was
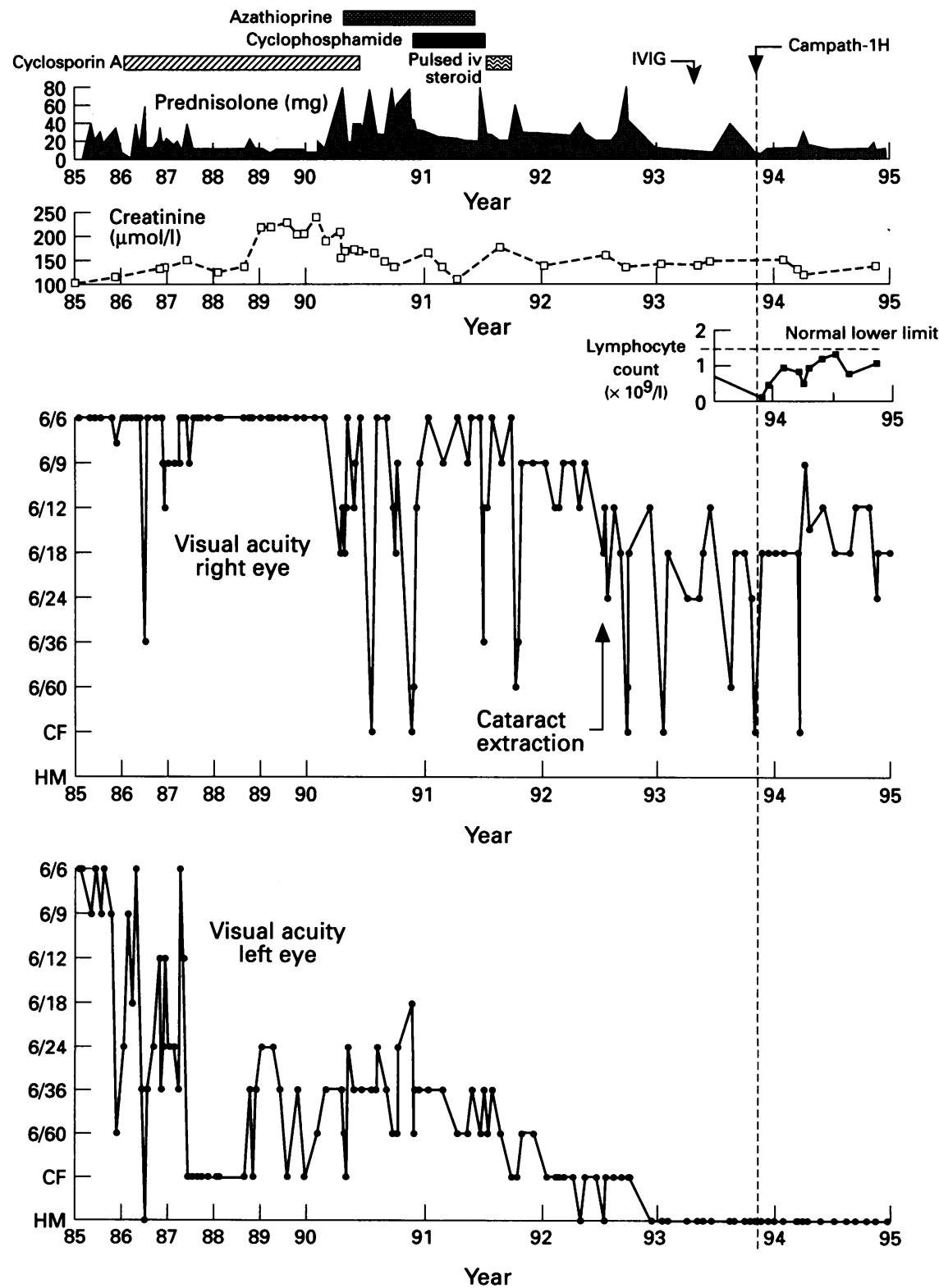

Figure 1 The clinical progress of the patient described. The lower two panels chart visual acuity, and the top panels chart immunosuppression, serum creatinine, and peripheral blood lymphocyte count. 